\title{
碱促进醛亚胺的快速氢硅化反应
}

\author{
王子超 ${ }^{a, b}$ 许佑君*,b施世良*,a \\ $\left({ }^{a}\right.$ 中国科学院上海有机化学研究所 金属有机化学国家重点实验室 上海 200032) \\ ( ${ }^{b}$ 沈阳药科大学 基于靶点的药物设计与研究教育部重点实验室 沈阳 110016)
}

\begin{abstract}
摘要 胺类化合物是一类重要的合成中间体, 亚胺的氢硅化反应为胺类化合物的合成提供了一种便利的方法. 过渡金 属及主族元素催化剂在亚胺氢硅化反应中得到了广泛应用, 然而, 采用更加简单的反应条件实现这类反应更加具有实 用价值. 利用廉价易得的叔丁醇钾作为促进剂, 实现了醛亚胺的快速还原反应, 以良好的收率高效合成了一系列胺类 化合物. 该反应体系具有反应迅速, 条件简单, 实用高效的优点.

关键词 氢硅化反应; 醛亚胺; 快速反应; 碱催化
\end{abstract}

\section{Base-Promoted Rapid Hydrosilylation of Aldimine}

\author{
Wang, Zichao ${ }^{a, b} \quad \mathrm{Xu}$, Youjun ${ }^{*, b} \quad$ Shi, Shiliang*,a
}

( ${ }^{a}$ State Key Laboratory of Organometallic Chemistry, Shanghai Institute of Organic Chemistry, Shanghai 200032)

$\left({ }^{b}\right.$ Key Laboratory of Structure-Based Drug Design \& Discovery, Shenyang Pharmaceutical University, Shenyang 110016)

\begin{abstract}
Amines are important intermediates in organic synthesis. The hydrosilylation of imines provides a convenient method for the synthesis of amines. Transition metal and main group catalysts have been widely applied in the hydrosilylation of imines. However, it would be more practical to use simpler reaction conditions to realize such reactions. In this paper, a fast hydrosilylation of aldimine was realized by using cheap $t$-BuOK as the promoter, providing an efficient method for the synthesis of amines in high yield. This reaction features rapid reaction, facile conditions, and robust practicability.

Keywords hydrosilylation; aldimine; rapid reaction; base catalysis
\end{abstract}

胺类化合物是一类重要的结构单元, 广泛存在于具 有生物活性的天然产物及药物分子中, 是药物合成中常 见的重要中间体 ${ }^{[1]}$, 且与人类健康息息相关, 如氨基酸、 维生素等结构中具有胺类片段. 因此, 合成化学家一直 致力于开发高效实用的方法来合成此类化合物. 目前, 传统的羰基化合物的还原胺化反应是合成这类化合物 最常用的方法 ${ }^{[2]}$, 通过金属催化的酰胺还原反应也可以 制备该类结构 ${ }^{[3]}$, 但由于酰胺羰基亲电性较弱, 这种方 法目前局限性较大. 此外, 亚胺的还原反应为这类化合 物的合成提供了另一种行之有效的方法, 还原方式主要 包括催化氢化 ${ }^{[4]}$ 、转移氢化 ${ }^{[5]}$ 、金属氢试剂还原及氢硅 化还原等, 其中, 硅烷作为还原剂具有安全、稳定、易 于处理、条件温和等特性, 近年来过渡金属催化的氢硅 化反应受到了化学家广泛的关注，一些贵金属如 $\mathrm{Rh}$ 、
$\mathrm{Ru} 、 \mathrm{Ir}$ 催化的氢硅化反应陆续被一些课题组所报道 ${ }^{[6]}$, 另外, $\mathrm{Fe}, \mathrm{Cu}, \mathrm{Ti}$ 等金属也可以催化此类反应顺利进行 ${ }^{[7]}$. 除了过渡金属, 研究者随后也发展了 $\mathrm{Al}$ 等主族金属参 与的亚胺氢硅化反应 ${ }^{\left[{ }^{[8]}\right.}$, 但反应实用性及底物适用性均 有待提高.

由于过渡金属催化剂的制备较复杂，且在药物合成 后期需要严格去除金属残留, 这些方法在药物生产中的 应用受到了一定的限制. 鉴于此, 化学家们随后发展了 许多无金属参与的亚胺氢硅化反应, 如硼酸、硅基离子 及亲电性膦离子也可顺利催化亚胺在温和条件下的还 原反应 ${ }^{[9]}$, 但这些方法需要特殊的催化剂, 而且适用范 围受限，其实用性有待提高. 因此，发展实用、高效的无 金属催化的亚胺氢硅化反应具有重要的研究价值. 最 近, 我们课题组 ${ }^{[10]}$ 报道了简单碱催化的烯烃及烯丙醇

* Corresponding authors. E-mail: shiliangshi@sioc.ac.cn; xuyoujun@syphu.edu.cn Received May 29, 2020; revised July 3, 2020; published online July 22, 2020. Dedicated to Professor Henry N. C. Wong on the occasion of his 70th birthday. Project supported by the Strategic Priority Research Program of the Chinese Academy of Sciences (No. XDB20000000) and the National Natural Science Foundation of China (Nos. 21690074, 21871288, 91856111, 21821002). 中国科学院战略性先导科技专项(No. XDB20000000)和国家自然科学基金(Nos. 21690074, 21871288, 91856111, 21821002)资助项目. 
的氢嗍化反应, 通过催化量的碱直接活化 $\mathrm{B}-\mathrm{H}$ 键, 实 现了高效的氢硼化过程. 在本课题组前期研究 ${ }^{[10]}$ 及前 人 $^{[11]}$ 研究的基础上, 本工作采用碱实现了亚胺化合物的 还原氢硅化反应, 无需外加金属催化剂, 条件简单. 值 得一提的是, 反应仅需 $10 \mathrm{~min}$ 即可完成, 为胺类化合物 的快速构建提供了一种简单实用的方法.

(A) Transition-metal-catalyzed hydrosilylation of aldimine

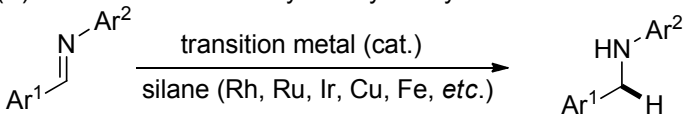

(B) Main-group-catalyzed hydrosilylation of aldimine

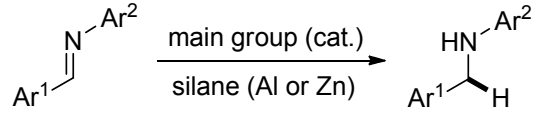

(C) Base-promoted hydrosilylation of aldimine (This work)

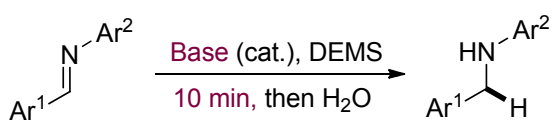

图式 1 亚胺氢硅化反应

Scheme 1 Hydrosilylation reaction of imine

\section{1 结果与讨论}

\section{1 反应条件优化}

选取 $N, 1$-二苯基甲酰胺(1a)和二乙氧基甲基硅烷 (DEMS，2)作为模板底物进行反应条件的优化, 具体实 验结果见表 1 . 在氮气氛围中, 以四氢呋喃(THF)为溶 剂, 在室温(r.t.)下对反应进行了初步尝试. 结果表明不 加碱时, 反应无法发生, 未检测到相应还原产物(表 1 , Entry 1). 受之前工作的启发, 我们设想加入催化量的碱 可能会与亲电性的硅烷生成高配位复合物, 通过活化 $\mathrm{Si}$ - $\mathrm{H}$ 键来促进相应的氢硅化过程. 为了验证这个设想, 随后在反应中加入催化量的碱进行尝试, 实验发现, 加 入 $5.0 \mathrm{mo} \%$ 催化量的叔丁醇钾 $(t-\mathrm{BuOK})$ 时, 反应 $2.0 \mathrm{~h}$ 后 几乎以定量的核磁收率得到目标产物(表 1, Entry 2). 考 察了不同种类的碱对反应的影响, 发现叔丁醇锂 $(t-\mathrm{BuOLi})$ 与氢氧化钠 $(\mathrm{NaOH})$ 均能催化反应进行 (表 1 , Entries 3,4), 但收率均有所下降, 这可能是由于碱性较 弱 $(t-\mathrm{BuOLi})$ 或溶解性较差 $(\mathrm{NaOH})$ 导致的. 加入弱碱(表 1, Entries 5 9), 如 $\mathrm{CsF}, \mathrm{K}_{3} \mathrm{PO}_{4}, \mathrm{KOAc}, \mathrm{Cs}_{2} \mathrm{CO}_{3}, \mathrm{PhONa}$, 反应效果均不理想, 对反应几乎没有促进作用, 因此最 终选择 $t$-BuOK 作为反应的促进剂. 紧接着, 将反应时 间缩短至 $10 \mathrm{~min}$, 实验发现反应仍能以定量的收率得到 产物(表 1, Entry 1), 因此相信这种亚胺快速还原的方式 对于胺类化合物的快速构建具有重要意义. 当反应体系 不加溶剂时, 反应仍得到定量还原产物(表 1, Entry 1), 但考虑到其他底物的溶解性差异, 最终仍选择 THF 作 为反应的溶剂. 基于上述优化结果, 确定了该反应的最 佳反应条件: $0.2 \mathrm{mmol} \mathrm{1a}, 2$ equiv. DEMS 为还原试剂,
$5.0 \mathrm{~mol} \% t-\mathrm{BuOK}$ 为碱, $\mathrm{THF}$ 为溶剂, 反应在室温下于氮 气氛围中反应 $10 \mathrm{~min}$. 反应最终可以 $99 \%$ 的收率得到亚 胺还原产物(表 1, Entry 10), 推测 DEMS 在催化量碱的 作用下可歧化为高活性氢硅化试剂 $\mathrm{MeSiH}_{3}$, 从而催化 亚胺还原 ${ }^{[11]}$.

表 1 反应条件的笁选和优化 ${ }^{a}$

Table 1 Optimization of the reaction conditions

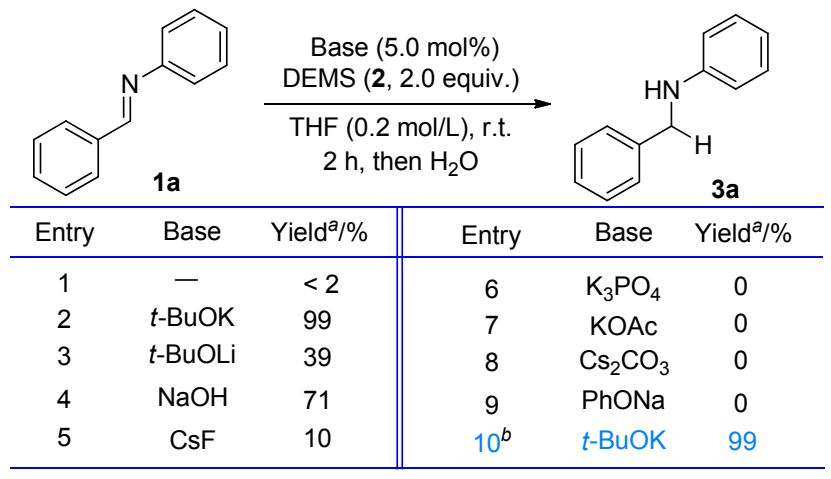

${ }^{a}$ Yields were determined by NMR analysis using 1,3,5-trimethyl-benzene as an internal standard. ${ }^{b} 10 \mathrm{~min}$, neat or THF $\left(1.0 \mathrm{~mol} \cdot \mathrm{L}^{-1}\right)$

\section{2 反应底物扩展}

确定了上述最优条件后, 采用 DEMS 为还原剂, 对 亚胺氢硅化反应的底物范围进行了简单研究. 具体结果 见表 2. 根据实验结果可以看出, 底物中的位阻作用对 于反应并没有影响，当使用含萗的底物 $(3 \mathbf{b})$, 或苯环的 邻位有甲基 $(3 c)$ 、甲氧基(3d)等位阻基团取代时，均能以 优秀的产率得到相应目标产物. 随后考察了苯环上不同 电性取代基对于反应的影响，发现芳环上含有供电子基 或吸电子基时，该体系均可适用 $(\mathbf{3 d} \sim 3 \mathbf{3})$. 在官能团耐 受性考察中，发现卤素(3e，3f)、甲硫基(3g)、三氟甲基 $(3 h) 、 三$ 氟甲氧基(3i)、炔基 $(3 j)$ 等基团均能兼容反应条 件. 尤其是，底物含有卤素和炔等官能团取代基时，反 应未受明显影响, 这为底物的进一步修饰提供了可能. 有意思的是，将氮上取代基更换为丁基或 Boc 保护基 时，或者使用烷基醛亚胺底物，即使在 $80{ }^{\circ} \mathrm{C}$ 加热过夜 条件下，几乎没有检测到产物生成，这可能是由于亚胺 底物的取代基电子或位阻效应而导致活性下降.

\section{3 克级反应研究}

为了说明反应的实用性, 对反应进行了克级规模的 研究. 如表 3 所示, 当把反应扩大至 $10 \mathrm{mmol}$ 时, 在无 溶剂条件下加入 $5.0 \mathrm{~mol} \%$ 的 $t$ - $\mathrm{BuOK}$, 室温反应 $10 \mathrm{~min}$, 能以 $97 \%$ 的收率得到产物 $(1.77 \mathrm{~g})$. 为了进一步增加反 应的实用性，采用价格更加低廉的 PMHS(聚甲基氢硅 烷)作为反应的还原剂(约 $800 Y / 250 \mathrm{~g}$ ), 在 $10 \mathrm{mmol}$ 规 模下，仍能以 $89 \%$ 的收率得到还原产物(1.62 g). 由于反 应迅速且条件简单，相信这种方法将在化合物库的快速 
表 2 底物拓展

Table 2 Scope of substrate

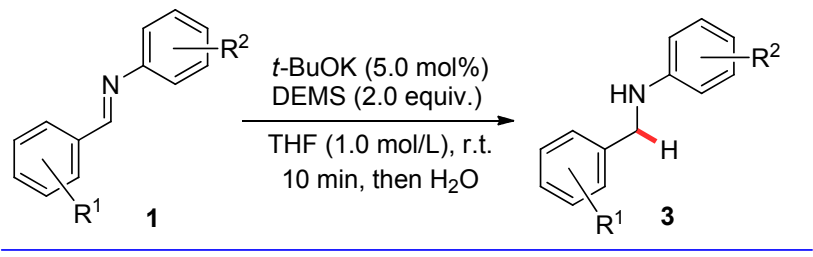

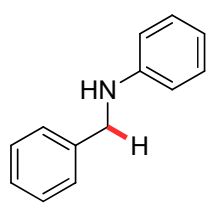

3a, $97 \%$ yield<smiles>COc1ccccc1CNc1ccccc1</smiles>

3d, $95 \%$ yield

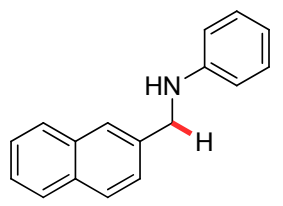

3b, $99 \%$ yield<smiles>Fc1ccc(CNc2ccccc2)cc1</smiles>

3 e, $92 \%$ yield

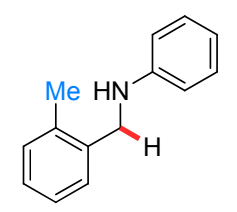

3c, $99 \%$ yield<smiles>CS(=O)(=O)c1ccc(CNc2ccccc2)cc1</smiles>

$\mathbf{3 g}, \mathbf{9 9 \%}$ yield<smiles>COc1ccc(NCc2ccc(OC(F)(F)F)cc2)cc1C(Nc1ccccc1)c1cc(C(F)(F)F)cc(C(F)(F)F)c1</smiles>

3h, $94 \%$ yield

$3 \mathbf{i}, 99 \%$ yield

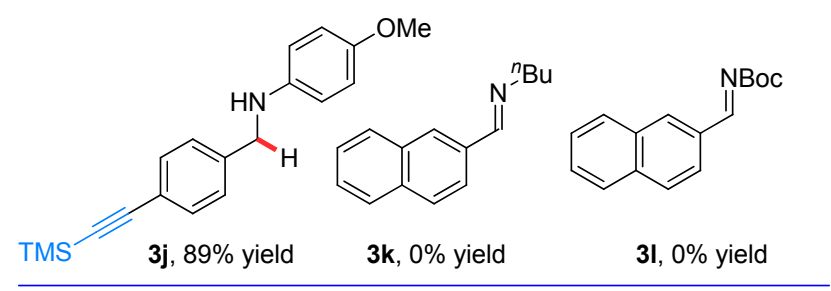<smiles>C(=N/c1ccccc1)\c1ccccc1</smiles>
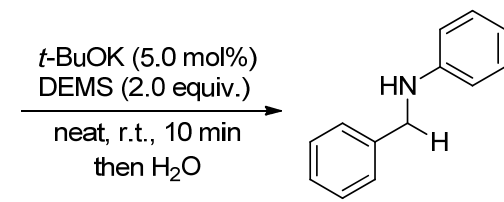

$10 \mathrm{mmol}$<smiles>C(=N/c1ccccc1)\c1ccccc1</smiles>

$10 \mathrm{mmol}$

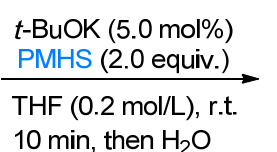
10 min, then $\mathrm{H}_{2} \mathrm{O}$

图式 2 克级反应

Scheme 2 Gram-scale reaction

构建中得到应用.

\section{2 结论}

本文报道了一种碱促进的快速氢硅化过程, 反应采
用廉价易得的硅烷作为还原剂, $10 \mathrm{~min}$ 即可高效地实现 醛亚胺的还原反应. 反应过程无需过渡金属参与, 反应 迅速，为一些胺类化合物的合成提供了一种简单实用的 方法.

\section{3 实验部分}

\section{1 仪器与试剂}

${ }^{1} \mathrm{H}$ NMR 和 ${ }^{13} \mathrm{C}$ NMR 在 $400 \mathrm{MHz}$ 核磁共振仪上测 定, $\mathrm{CDCl}_{3}$ 为溶剂, TMS 为内标. 所用试剂均为分析纯或 化学纯, 使用前未经纯化.

\section{2 实验方法}

在氮气保护范围下, 于干燥的反应瓶中依次加入醛 亚胺 $(1,0.2 \mathrm{mmol})$, THF $\left(0.2 \mathrm{~mL}, 1.0 \mathrm{~mol} \cdot \mathrm{L}^{-1}\right)$, DEMS (2, $0.2 \mathrm{mmol}, 2$ equiv.), $t$-BuOK (5.0 mol\%, $1.1 \mathrm{mg}$ ), 反应混 合物在室温下搅拌反应 $10 \mathrm{~min}$. 反应结束后, 加水淬灭 反应, 加入乙酸乙酯萃取, 有机层用饱和氯化钠溶液洗 涤，无水硫酸钠干燥，除去干燥剂，减压浓缩，柱层析 色谱分离得纯化合物 $\mathbf{3}$.

$\mathrm{N}$-苄基苯胺 (3a $)^{[12 \mathrm{a}]}$ : $35.6 \mathrm{mg}$, 无色油状物, 产率 97\%. ${ }^{1} \mathrm{H}$ NMR (400 MHz, $\left.\mathrm{CDCl}_{3}\right) \delta: 7.42 \sim 7.28(\mathrm{~m}, 4 \mathrm{H})$, $7.28 \sim 7.21(\mathrm{~m}, 1 \mathrm{H}), 7.20 \sim 7.08(\mathrm{~m}, 2 \mathrm{H}), 6.76 \sim 6.67(\mathrm{~m}$, $1 \mathrm{H}), 6.60 \sim 6.61(\mathrm{~m}, 2 \mathrm{H}), 4.28(\mathrm{~s}, 2 \mathrm{H}), 3.97(\mathrm{~s}, 1 \mathrm{H}) ;{ }^{13} \mathrm{C}$ NMR $\left(101 \mathrm{MHz}, \mathrm{CDCl}_{3}\right) \delta: 148.2,139.5,129.4,128.7$, 127.6, 127.3, 117.6, 112.9, 48.4 .

$N$-(萗-2-基甲基)苯胺 $(\mathbf{3 b})^{[12 b]}: 46.5 \mathrm{mg}$, 无色油状物, 产率 99\%. ${ }^{1} \mathrm{H}$ NMR $\left(400 \mathrm{MHz}, \mathrm{CDCl}_{3}\right) \delta: 8.00 \sim 7.65(\mathrm{~m}$, $4 \mathrm{H}), 7.52 \sim 7.34(\mathrm{~m}, 3 \mathrm{H}), 7.24 \sim 7.05(\mathrm{~m}, 2 \mathrm{H}), 6.68 \sim 6.72$ (m, 1H), $6.65 \sim 6.55(\mathrm{~m}, 2 \mathrm{H}), 4.41(\mathrm{~s}, 2 \mathrm{H}) ;{ }^{13} \mathrm{C}$ NMR $(101$ $\left.\mathrm{MHz}, \mathrm{CDCl}_{3}\right) \delta: 148.3,137.1,133.6,132.8,129.4,128.5$, $127.9,127.8,126.3,126.0,125.9,125.8,117.7,113.0$, 48.5.

$N$-(2-甲基苄基)苯胺(3c $)^{[12 \mathrm{c}]}$ : $39.0 \mathrm{mg}$, 无色油状物, 产率 $99 \% .{ }^{1} \mathrm{H}$ NMR $\left(400 \mathrm{MHz}, \mathrm{CDCl}_{3}\right) \delta: 7.44 \sim 7.36(\mathrm{~m}$, $1 \mathrm{H}), 7.30 \sim 7.20(\mathrm{~m}, 5 \mathrm{H}), 6.76 \sim 6.80(\mathrm{~m}, 1 \mathrm{H}), 6.74 \sim 6.65$ (m, 2H), 4.32 (s, 2H), 2.43 (s, 3H); ${ }^{13} \mathrm{C}$ NMR (101 MHz, $\left.\mathrm{CDCl}_{3}\right) \delta: 148.4,137.1,136.5,130.5,129.4,128.4,127.5$, 126.3, 117.6, 112.8, 46.5, 19.1.

$\mathrm{N}$-(2-甲氧基苄基)苯胺 (3d $)^{[12 \mathrm{~d}]}: 40.5 \mathrm{mg}$, 无色油状 物, 产率 95\%. ${ }^{1} \mathrm{H}$ NMR $\left(400 \mathrm{MHz}, \mathrm{CDCl}_{3}\right) \delta: 7.28(\mathrm{~d}, J=$ $8.8 \mathrm{~Hz}, 2 \mathrm{H}), 7.21 \sim 7.11(\mathrm{~m}, 2 \mathrm{H}), 6.87(\mathrm{~d}, J=8.8 \mathrm{~Hz}, 2 \mathrm{H})$, $6.74 \sim 6.67(\mathrm{~m}, 1 \mathrm{H}), 6.61 \sim 6.63(\mathrm{~m}, 2 \mathrm{H}), 4.23(\mathrm{~s}, 2 \mathrm{H})$, 3.79 (s, 3H); ${ }^{13} \mathrm{C}$ NMR $\left(101 \mathrm{MHz}, \mathrm{CDCl}_{3}\right) \delta: 158.9,148.3$, 131.5, 129.4, 128.9, 117.6, 114.1, 112.9, 55.4, 55.4, 47.9 .

$N$-(4-氟苠基)苯胺 $(\mathbf{3 e})^{[12 \mathrm{~d}]}$ : $37.0 \mathrm{mg}$, 无色油状物, 产率 $92 \% .{ }^{1} \mathrm{H}$ NMR $\left(400 \mathrm{MHz}, \mathrm{CDCl}_{3}\right) \delta: 7.30 \sim 7.34(\mathrm{~m}$, 
2H), 7.17 (t, $J=8.0 \mathrm{~Hz}, 2 \mathrm{H}), 7.01(\mathrm{t}, J=8.7 \mathrm{~Hz}, 2 \mathrm{H}), 6.72$ $(\mathrm{t}, J=7.3 \mathrm{~Hz}, 1 \mathrm{H}), 6.61(\mathrm{~d}, J=7.5 \mathrm{~Hz}, 2 \mathrm{H}), 4.29(\mathrm{~s}, 2 \mathrm{H})$, $4.00(\mathrm{~s}, 1 \mathrm{H}) ;{ }^{13} \mathrm{C}$ NMR $\left(101 \mathrm{MHz}, \mathrm{CDCl}_{3}\right) \delta: 162.1$ (d, $J=$ $246.44 \mathrm{~Hz}$ ), 147.9, 135.2 (d, $J=3.3 \mathrm{~Hz}$ ), 129.3, 129.0 (d, $J=8.1 \mathrm{~Hz}), 117.8,115.5(\mathrm{~d}, J=22.2 \mathrm{~Hz}), 112.9,47.6$.

$N$-(4-氯苄基)苯胺 $(\mathbf{3 f})^{[12 \mathrm{a}]}: 40.0 \mathrm{mg}$, 无色油状物, 产 率 92\%. ${ }^{1} \mathrm{H}$ NMR (400 MHz, $\mathrm{CDCl}_{3}$ ) $\delta: 7.29$ (s, 4H), 7.17 $(\mathrm{d}, J=15.8 \mathrm{~Hz}, 2 \mathrm{H}), 6.72(\mathrm{t}, J=7.3 \mathrm{~Hz}, 1 \mathrm{H}), 6.60(\mathrm{~d}, J=$ $8.1 \mathrm{~Hz}, 2 \mathrm{H}), 4.30$ (s, 2H), $4.05(\mathrm{~s}, 1 \mathrm{H}) ;{ }^{13} \mathrm{C}$ NMR $(101$ $\left.\mathrm{MHz}, \mathrm{CDCl}_{3}\right) \delta: 147.9,138.1,132.9,129.4,128.9,128.8$, $117.9,112.9,47.7$.

$\mathrm{N}$-(4-(甲硫基)苠基)苯胺(3g) ${ }^{[12 \mathrm{e}]}: 45.5 \mathrm{mg}$, 无色油状 物, 产率 99\%. ${ }^{1} \mathrm{H}$ NMR (400 MHz, $\left.\mathrm{CDCl}_{3}\right) \delta: 7.28(\mathrm{~d}, J=$ $8.3 \mathrm{~Hz}, 2 \mathrm{H}), 7.22$ (d, $J=8.4 \mathrm{~Hz}, 2 \mathrm{H}), 7.16$ (t, $J=8.0 \mathrm{~Hz}$, 2H), $6.71(\mathrm{t}, J=7.4 \mathrm{~Hz}, 1 \mathrm{H}), 6.61(\mathrm{~d}, J=7.5 \mathrm{~Hz}, 2 \mathrm{H}), 4.27$ (s, 2H), 3.99 (s, 1H), $2.46(\mathrm{~s}, 3 \mathrm{H}) ;{ }^{13} \mathrm{C} \mathrm{NMR}(101 \mathrm{MHz}$, $\left.\mathrm{CDCl}_{3}\right) \delta: 148.2,137.3,136.5,129.4,128.1,127.1,117.7$, $112.9,47.9,16.2$.

$N$-(3,5-二(三氟甲基)苄基)苯胺(3h)：60.0 mg, 无色 油状物, 产率 94\%. ${ }^{1} \mathrm{H}$ NMR (400 $\mathrm{MHz}^{\left.-\mathrm{CDCl}_{3}\right)} \delta: 7.84$ (s, 2H), $7.79(\mathrm{~s}, 1 \mathrm{H}), 7.19(\mathrm{t}, J=8.0 \mathrm{~Hz}, 2 \mathrm{H}), 6.77(\mathrm{t}, J=$ $7.4 \mathrm{~Hz}, 1 \mathrm{H}), 6.60$ (d, $J=7.6 \mathrm{~Hz}, 2 \mathrm{H}), 4.47$ (s, 2H), 4.18 (s, $1 \mathrm{H}) ;{ }^{13} \mathrm{C} \mathrm{NMR}\left(101 \mathrm{MHz}, \mathrm{CDCl}_{3}\right) \delta: 147.4,142.7,132.1$ (q, $J=33.3 \mathrm{~Hz}), 129.6,127.4$ (m), 124.8, 122.1, 121.4 (m), 118.7, 113.2, 47.9; ${ }^{19} \mathrm{~F}$ NMR $\left(376 \mathrm{MHz}, \mathrm{CDCl}_{3}\right) \delta$ : 62.82; HRMS (ESI) calcd for $\mathrm{C}_{15} \mathrm{H}_{12} \mathrm{~F}_{6} \mathrm{~N}[\mathrm{M}+\mathrm{H}]^{+}$ 320.0869 , found 320.0870 .

4-甲基- $N$-(4-(三氟甲氧基)苠基)苯胺(3i)：59.5 mg, 无色油状物, 产率 99\%. ${ }^{1} \mathrm{H}$ NMR $\left(400 \mathrm{MHz} \mathrm{CDCl}_{3}\right) \delta$ : 7.39 (d, $J=8.5 \mathrm{~Hz}, 2 \mathrm{H}), 7.17$ (d, $J=8.3 \mathrm{~Hz}, 2 \mathrm{H}), 6.77$ (d, $J=8.9 \mathrm{~Hz}, 2 \mathrm{H}), 6.58$ (d, $J=8.9 \mathrm{~Hz}, 2 \mathrm{H}), 4.29$ (s, 2H), 3.73 $(\mathrm{s}, 3 \mathrm{H}) ;{ }^{13} \mathrm{C}$ NMR $\left(101 \mathrm{MHz}, \mathrm{CDCl}_{3}\right) \delta: 152.5,148.4$, 142.2, 138.6, 128.8, 121.3, 120.6 (q, $J=252.5 \mathrm{~Hz}$ ), 115.0, 114.3, 55.9, 48.6; ${ }^{19} \mathrm{~F}$ NMR (376 $\left.\mathrm{MHz} \mathrm{CDCl}_{3}\right) \delta$ : 57.89; HRMS (ESI) calcd for $\mathrm{C}_{15} \mathrm{H}_{13} \mathrm{NO}_{2} \mathrm{~F}_{3}[\mathrm{M}-\mathrm{H}]^{+}$ 296.0893, found 296.0894..

4-甲氧基- $N$-(4-((三甲硅基)乙炔基)苠基)苯胺 $(3 \mathbf{j})$ : $55.0 \mathrm{mg}$, 无色油状物, 产率 89\%. ${ }^{1} \mathrm{H}$ NMR (400 MHz, $\left.\mathrm{CDCl}_{3}\right) \delta: 7.43(\mathrm{~d}, J=8.1 \mathrm{~Hz}, 2 \mathrm{H}), 7.29(\mathrm{~d}, J=8.1 \mathrm{~Hz}$, $2 \mathrm{H}), 6.75$ (d, $J=8.9 \mathrm{~Hz}, 2 \mathrm{H}), 6.56$ (d, $J=9.0 \mathrm{~Hz}, 2 \mathrm{H}), 4.27$ (s, 2H), 3.73 (s, 3H), $0.24(\mathrm{~s}, 9 \mathrm{H}) ;{ }^{13} \mathrm{C}$ NMR (101 MHz, $\left.\mathrm{CDCl}_{3}\right) \delta: 152.4,142.3,140.4,132.3,127.4$ 121.9, 114.9, 114.3, 105.1, 94.1, 55.9, 49.1, 0.1; HRMS (ESI) calcd for $\mathrm{C}_{19} \mathrm{H}_{22} \mathrm{NOSi}[\mathrm{M}-\mathrm{H}]^{+}$308.1465, found 308.1456.
辅助材料(Supporting Information) 所有目标化合物 的核磁共振氢谱、碳谱和氟谱. 这些材料可以免费从本 刊网站(http://sioc-journal.cn/)上下载.

\section{References}

[1] (a) Lawrence, S. A. Amines: Synthesis, Properties and Applications, Cambridge University, Press, Cambridge, 2004.

(b) Ricci, A. Amino Group Chemistry: Synthesis to the Life Sciences, Wiley-VCH, Weinheim, 2008.

[2] Nugent, T. C.; El-Shazly, M. Adv. Synth. Catal. 2010, 352, 753.

[3] (a) Das, S.; Wendt, B.; Möller, K.; Junge, K.; Beller, M. Angew. Chem., Int. Ed. 2012, 51, 1662.

(b) Das, S.; Li, Y.; Bornschein, C.; Pisiewicz, S.; Kiersch, K.; Michalik, D.; Gallou, F.; Junge, K.; Beller, M. Angew. Chem., Int. Ed. 2015, 54, 12389.

[4] (a) Spindler, F. Handbook of Homogeneous Hydrogention, Wiley-VCH, Weinheim, 2007.

(b) Hu, X.; Hu, F.; Zhang, M.; Liao, Y.; Xu, X.; Yuan, W.; Zhang, X. Chin. J. Org. Chem. 2016, 36, 1895 (in Chinese).

(扈晓艳, 胡方芝, 张敏敏, 廖益均, 徐小英, 袁伟成, 张晓梅, 有机化学, 2016, 36, 1895.)

(c) Wang, D.; Hou, C.; Chen, L.; Liu, X.; An, Q.; Hu, X. Chin. J. Org. Chem. 2013, 33, 1355 (in Chinese).

(王东, 侯传金, 陈丽风, 刘小宁, 安庆大, 胡向平, 有机化学, 2013, 33, 1355.)

[5] Zhu, C.; Saito, K.; Yamanaka, M.; Akiyama, T. Acc. Chem. Res. $\mathbf{2 0 1 5}, 48,388$

[6] (a) Nishibayashi, Y.; Takei, I.; Uemura, S.; Hidai, M. Organometallics 1998, 17, 3420

(b) Takei, I.; Nishibayashi, Y.; Arikawa, Y.; Uemura, S.; Hidai, M. Organometallics 1999, 18, 2271.

[7] (a) Saini, A.; Smith, C. R.; Wekesa, F. S.; Helm, A. K.; Findlater, M. Org. Biomol. Chem., 2018, 16, 9368.

(b) Lipshutz, B. H.; Shimizu, H. Angew. Chem. Int. Ed. 2004, 43, 2228.

(c) Hansen, M. C.; Buchwald, S. L. Org. Lett., 2000, 2, 713.

[8] (a) Koller, J.; Bergman, R. G. Organometallics 2012, 31, 2530.

(b) Bezada, A.; Szewczyk, M.; Mlynarski, J. J. Org. Chem. 2016, 81,336

[9] (a) Chardon, A.; Rouden, J.; Blanchet, J. Eur. J. Org. Chem. 2019, 995.

(b) Prez, M.; Qu, Z.-W.; Caputo, C. B.; Podgorny, V.; Hounjet, L. J.; Hansen, A.; Dobrovetsky, R.; Grimme, S.; Stephan, D. W. Chem.-Eur. J. 2015, 21, 1 .

(c) Müther, K.; Mohr, J.; Oestreich, M. Organometallics 2013, 32, 6643.

(d) Ge, X.; Chen, X.; Qian, C. Chin. J. Org. Chem. 2016, 36, 1208 (in Chinese)

(葛新，陈新志，钱超，有机化学, 2016, 36, 1208.)

[10] (a) Wang, Z.-C.; Shen, D.; Gao, J. Jia, X.; Xu, Y.; Shi, S.-L. Chem. Commun. 2019, 55, 8848.

(b) Wang, Z.-C.; Wang, M.; Gao, J.; Shi, S.-L.; Xu Y. Org. Chem. Front. 2019, 6, 2949.

[11] Revunova, K.; Nikonov, G. I. Chem.-Eur. J. 2014, $20,839$.

[12] (a) Bagal, D. B; Watile, R. A; Khedkar, M. V.; Dhake, K. P.; Bhanage, B. M. Catal. Sci. Technol. 2012, 2, 354.

(b) Lee, C. C.; Liu, S. T. Chem. Commun. 2011, 47, 6981.

(c) Ohta, H.; Yuyama, Y.; Uozumi, Y.; Yamada, Y. M. A. Org. Lett. 2011, 13, 3892.

(d) Zhan, M.; Yang, H.; Zhang, Y.; Zhu, C.; Li, W.; Cheng, Y.; Hua, H. Chem. Commun. 2011, 47, 6605.

(e) Sousa, S. C. A.; Fernandes, A. C. Adv. Synth. Catal. 2010, 352, 2218. 